\title{
Inferring rheology and geometry of subsurface structures by adjoint-based inversion of principal stress directions
}

\author{
G.S. Reuber ${ }^{1,2 \star}$, L. Holbach ${ }^{3}$, A.A. Popov ${ }^{1}$, M. Hanke ${ }^{3,4,5}$, B.J.P. Kaus ${ }^{1,4,5}$ \\ ${ }^{1}$ Institute of Geosciences, Johannes Gutenberg-University, 55128 Mainz, Germany \\ ${ }^{2}$ Max-Planck Graduate Center, Johannes Gutenberg-University, 55128 Mainz, Germany \\ ${ }^{3}$ Institute of Mathematics, Johannes Gutenberg-University, 55128 Mainz, Germany \\ ${ }^{4}$ Computational Sciences Mainz, Johannes Gutenberg-University, 55128 Mainz, Germany \\ ${ }^{5}$ Mainz Institute of Multiscale Modelling, Johannes Gutenberg-University, 55128 Mainz, Germany
}

\section{SUMMARY}

Imaging subsurface structures, such as salt domes, magma reservoirs, or subducting plates, is a major challenge in geophysics. Seismic imaging methods are, so far, the most precise methods to open a window into the Earth. However, the methods may not yield the exact depth or size of the imaged feature and may become distorted by phenomena such as seismic anisotropy, fluid flow, or compositional variations. A useful complementary method is therefore to simulate the mechanical behavior of rocks on large timescales, and compare model predictions with observations. Recent studies have used the (nonlinear) Stokes equations and geometries from seismic studies in combination with an adjoint-based approach to invert for rheological parameters that are consistent with surface observations such as GPS velocities. Nevertheless, it would be useful to employ other surface observations, such as principal stress directions, as constraints as well. Here, we derive the adjoint formulation for the case that principal stress directions are used as observables with respect to rheological parameters. Both an algebraic and a discretized 


\section{G.S. Reuber}

derivation of the adjoint equations are described. This thus enables the usage of two data fields - surface velocities and stress directions - as a misfit for the inversion. We test the performance of the inversion for principal stress directions on simplified 3D test cases.

Finally, we demonstrate how the adjoint approach can be employed to compute 3D geodynamic sensitivity kernels, which highlight the areas in the model domain that have the largest impact on the misfit value of a particular point. This provides a simple, yet powerful, way to visualize which parts of the model domain are of key importance if changing rheological constants.

Key words: Numerical modeling - Inverse theory - Mechanics, theory, and modeling

\section{INTRODUCTION}

Geodynamic inverse methods aim to reproduce the shape (e.g., Worthen et al. 2014) and the material parameters (e.g., Reuber et al. 2018) of subsurface structures in order to fit data, such as plate velocities or principal stress directions, measured on or close to the Earth's surface. These measurements are usually expensive to obtain and cover the area of the Earth only sparsely. Inferring subsurface structures and material parameters merely from surface observations is thus a significant challenge. Additionally, considering the slow movement of plates, the measurements only represent instantaneous snapshots. It is thus beneficial to investigate the effect and the computational effort for incorporating as many observations as possible into the inverse problem. While sampling-based methods can take several data fields into account without changing the underlying equations (Baumann et al. 2014), they are computationally expensive as the number of required forward simulations scales in a nonlinear manner with the number of parameters to be inverted for, which makes them impractical for a large amount of unknown parameters. Gradient-based methods can be more efficient, particularly if the initial guess is close to the true solution and when combined with an adjoint approach to compute the gradient of the misfit function with respect to the material parameters. Yet, so far, the adjoint equations for the Stokes problems have only been derived for cases in which the misfit is given in velocities, but it has remained unclear how to incorporate other (derived) parameters such as stress. Here, we present an adjoint-based framework for efficient computation of gradients with principal stress directions as data, extending the known approach using surface velocities (e.g. Ismail-Zadeh et al. 2003). The adjoint method is widely used in seismology (e.g. Tarantola 2005; Tromp et al. 2005; Fichtner 2011) and has previously been applied to geodynamic problems (e.g. Ismail-Zadeh et al. 2003; Horbach et al. 2014; Ratnaswamy et al. 2015). However, the geodynamic applications were limited to global scale mantle convection problems to fit global plate velocities. In this work, we extend the derivation in order to include principal stress directions, which might come from measurements in boreholes or focal mechanism data determined from

* Corresponding author 
earthquakes, as data in the inverse framework. We only consider simplified problems in the numerical computations to build the basis for the future use of this method in larger frameworks and real world applications.

\section{GOVERNING EQUATIONS}

There is clear evidence from both geological observations and laboratory experiments that the slow deformation of rocks in the Earth's mantle and large parts of the lithosphere can be modeled as a highly viscous shear-thinning fluid. Motion in the Earth's mantle is thus usually described by the nonlinear Stokes equations stated in the following.

\subsection{Forward problem}

The instantaneous Stokes equations for incompressible fluids consist of conservation of mass and momentum on the domain $\Omega$ :

$$
\begin{array}{r}
\nabla \cdot \mathbf{u}=0, \\
\nabla \cdot \boldsymbol{\sigma}+\rho \mathbf{g}=\mathbf{0},
\end{array}
$$

where $\mathbf{u}=\left(u_{x}, u_{y}, u_{z}\right)^{T}$ is the velocity vector, $\boldsymbol{\sigma}$ is the Cauchy stress tensor, $\rho$ is the density, and $\mathbf{g}$ is the gravity acceleration vector. The deviatoric-volumetric stress decomposition is given by

$$
\boldsymbol{\sigma}=\boldsymbol{\tau}-p \mathbf{I}
$$

Here $\tau$ stands for the Cauchy stress deviator, $p$ is the pressure (positive in compression), and $\mathbf{I}$ is the second-order identity tensor.

We assume the constitutive equation for the deviatoric stress tensor in a standard power-law form:

$$
\boldsymbol{\tau}(\mathbf{u})=2 \eta(\mathbf{u}) \dot{\varepsilon}(\mathbf{u})
$$

where $\dot{\varepsilon}(\mathbf{u})=\frac{1}{2}\left(\nabla \mathbf{u}+(\nabla \mathbf{u})^{T}\right)$ is the strain rate tensor, and $\eta$ is the effective viscosity given by

$$
\eta(\mathbf{u})=\eta_{0}\left(\frac{\dot{\varepsilon}_{\mathrm{II}}(\mathbf{u})}{\dot{\varepsilon}_{0}}\right)^{\frac{1}{n}-1} .
$$

Here, $\eta_{0}$ is the reference viscosity, $\dot{\varepsilon}_{0}$ is the reference strain rate, $n$ is the power-law exponent, and $\dot{\varepsilon}_{\mathrm{II}}(\mathbf{u})=\sqrt{\frac{1}{2} \dot{\varepsilon}(\mathbf{u}): \dot{\varepsilon}(\mathbf{u})}$ is the effective strain rate (square root of the second invariant of the strain rate tensor).

To complete the formulation of the problem, we impose a free slip condition on the boundary $\partial \Omega$ of $\Omega$ :

$$
\begin{array}{r}
\mathbf{u} \cdot \mathbf{n}=0, \\
\mathbf{T}(\boldsymbol{\sigma} \mathbf{n})=\mathbf{0},
\end{array}
$$

where $\mathbf{n}$ is the outward normal vector and $\mathbf{T}=\mathbf{I}-\mathbf{n} \otimes \mathbf{n}$ is the tangential projection.

Equations (1) and (2) represent a formulation of the Stokes problem in a so-called strong, or differential, form. We found it more 


\section{G.S. Reuber}

convenient to utilize an equivalent weak, or integral, formulation so as to obtain a numerical solution of the forward problem. This can be achieved by using the standard two-step Galerkin procedure: (i) multiply equations (1) and (2) with the test functions $\mathbf{v}$ (for the velocity) and $q$ (for the pressure), where $\mathbf{v}$ satisfies the essential boundary condition (6), (ii) integrate over the domain and apply Green's first identity. The resulting formulation is: Find $\mathbf{u}$ and $p$ such that

$$
\begin{array}{r}
\int_{\Omega} 2 \eta(\mathbf{u}) \dot{\varepsilon}(\mathbf{u}): \dot{\varepsilon}(\mathbf{v}) d \mathbf{x} \\
-\int_{\Omega} p \nabla \cdot \mathbf{v} d \mathbf{x} \\
-\int_{\Omega} q \nabla \cdot \mathbf{u} d \mathbf{x} \\
-\int_{\Omega} \rho \mathbf{g} \cdot \mathbf{v} d \mathbf{x} \\
=0
\end{array}
$$

for all test functions $\mathbf{v}$ and $q$. Note that the spaces for the velocity and pressure ( $\mathbf{u}$ and $p$ ), and corresponding test functions ( $\mathbf{v}$ and $q$ ) are properly defined to enable combining the weak form into a single equation. The boundary integral in equation (8) is not present because of the free slip boundary conditions (6) and (7). The weak form of the Stokes problem (8) is directly amenable to discretization and solution by, e.g., the finite element method.

\subsection{Inverse problem with principal stress directions}

Although the effective physical and rheological parameters of the lithosphere and mantle (e.g., $\rho, \eta_{0}, n$ ) cannot generally be measured directly, they can still be estimated by solving an inverse problem. A geodynamic inversion can be formulated as a constrained optimization problem (see e.g., Ismail-Zadeh et al. 2003; Ratnaswamy et al. 2015; Reuber et al. 2017) where the difference between the observed data and simulation results is to be minimized, under the constraint that the Stokes equations - conservation of mass and momentum - are satisfied.

A common type of observation used in geodynamic inversions is the surface plate velocity field. However, there is an important pool of data that has so far not been incorporated in geodynamic inversions, namely stresses. In practice, the absolute values of the stress tensor are difficult to measure but it is easier to constrain the principal stress directions (PSD) from either borehole breakouts or seismic focal mechanism inversions (see e.g. Zoback 2010). A typical assumption that is made about the orientation of principal stresses in the lithosphere is a so-called Anderson's model illustrated in Fig. 1. One of the principal stresses is assumed to be vertical $\left(S_{\mathrm{v}}\right)$, and the other two are horizontal ( $S_{\mathrm{Hmin}}$ and $\left.S_{\mathrm{Hmax}}\right)$. The magnitudes of the principal stresses are rarely available, and the only parameter that can be used as a constraint for the geodynamic inversion is the orientation of the maximum horizontal compressive stress $\left(S_{\mathrm{Hmax}}\right)$ denoted as $\varphi$ in Fig. 1 . Only the direction of the principal stress can be constrained and so the angle is constrained to vary within the range $\varphi \in(0,180)^{\circ}$.

Assuming Anderson's model, the orientation angle of the maximum horizontal compressive stress can be computed using three compo- 


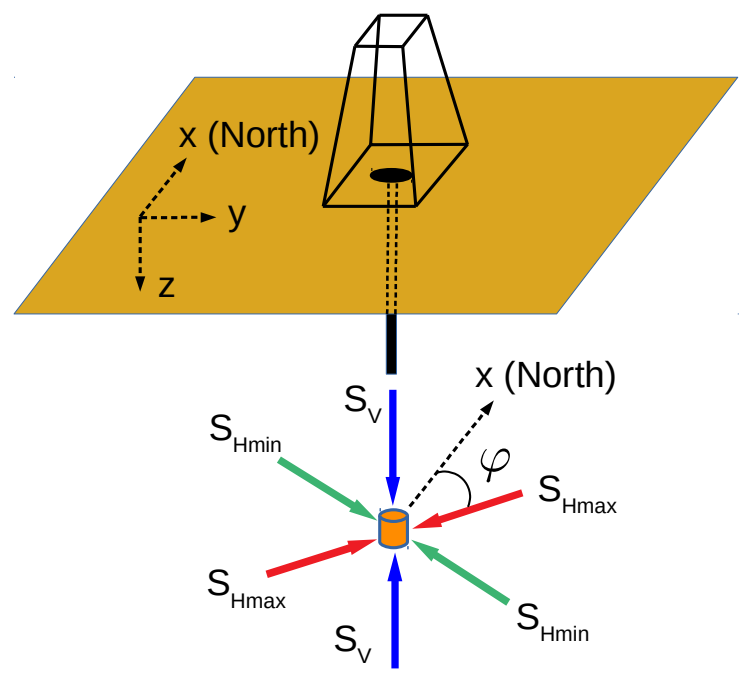

Figure 1. Principal stress orientation around a borehole according to Anderson's assumption. One of the principal stresses is assumed to be vertical $\left(S_{\mathrm{v}}\right)$, and the other two are horizontal $\left(S_{\mathrm{Hmin}}\right.$ and $\left.S_{\mathrm{Hmax}}\right)$. The angle $\varphi$ describes the direction of maximum horizontal compressive stress $\left(S_{\mathrm{Hmax}}\right)$ measured from the North.

nents of the deviatoric stress tensor (e.g. Gere 2004):

$$
\varphi=\frac{1}{2} \tan ^{-1}\left(\frac{2 \tau_{x y}}{\tau_{x x}-\tau_{y y}}\right)
$$

For the power-law viscous rheology with isotropic constitutive properties employed in this study it is possible to express the orientation angle in terms of the strain rate tensor, since it has the same unit direction, i.e.,

$$
\varphi=\frac{1}{2} \tan ^{-1}\left(\frac{2 \dot{\varepsilon}_{x y}}{\dot{\varepsilon}_{x x}-\dot{\varepsilon}_{y y}}\right)
$$

Note that for an elastic, rather than a viscous, rheology, the stress history becomes important and the deviatoric stress tensor deviates from the unit direction of the strain rate. A similar formulation for this case is possible, however, we leave it as a topic for a follow-up study.

Here, we extend the geodynamic inversion framework to incorporate PSD as a constraint. We formulate the inverse problem as a constrained infinite-dimensional nonlinear least squares optimization problem:

$$
\min _{\rho, \eta_{0}, n} \mathcal{F}\left(\rho, \eta_{0}, n\right)
$$

with a cost function defined by

$$
\mathcal{F}\left(\rho, \eta_{0}, n\right):=\frac{1}{2} \int_{\Omega_{\mathrm{obs}}}\left(\varphi-\varphi_{\mathrm{obs}}\right)^{2} d \mathbf{x}
$$

where $\varphi_{\text {obs }}$ denotes the stress directions measured on $\Omega_{\text {obs }} \subset \Omega$ and $\varphi$ is given by (10) through the solution of the nonlinear forward Stokes problem (8). In this approach, the material parameters $\rho, \eta_{0}$, and $n$ do not necessarily need to be treated as phase-based constants but can be considered as spatially varying parameter fields $\mathbf{m}(\mathbf{x})=\left(\rho(\mathbf{x}), \eta_{0}(\mathbf{x}), n(\mathbf{x})\right)^{T}$. To minimize the cost function $\mathcal{F}$, we employ a quasi-Newton method that requires computation of the gradient $\mathcal{G}(\mathbf{m})$ of $\mathcal{F}(\mathbf{m})$. 


\section{G.S. Reuber}

\subsection{Adjoint-based gradients}

The adjoint method is an efficient way to compute gradients for optimization problems with partial differential equations as constraints.

To derive these adjoint-based gradients, we will use the formal Lagrangian method (see Bertsekas 2014; Tröltzsch 2010). This requires the definition of the so-called Lagrangian functional and the computation of its derivatives. The Lagrangian functional is obtained by adding the cost function to the weak form of the forward problem and takes the following form for our framework:

$$
\begin{aligned}
\mathcal{L}(\mathbf{u}, p ; \mathbf{v}, q ; \mathbf{m})= & \frac{1}{2} \int_{\Omega_{\mathrm{obs}}}\left(\varphi-\varphi_{\mathrm{obs}}\right)^{2} d \mathbf{x} \\
& +\int_{\Omega} 2 \eta(\mathbf{u}) \dot{\varepsilon}(\mathbf{u}): \dot{\varepsilon}(\mathbf{v}) d \mathbf{x} \\
& -\int_{\Omega} p \nabla \cdot \mathbf{v} d \mathbf{x} \\
& -\int_{\Omega} q \nabla \cdot \mathbf{u} d \mathbf{x} \\
& -\int_{\Omega} \rho \mathbf{g} \cdot \mathbf{v} d \mathbf{x} .
\end{aligned}
$$

Here, the test functions $\mathbf{v}$ and $q$ become the adjoint velocity and adjoint pressure, respectively.

In the formal Lagrangian approach, the forward problem solution $\mathbf{u}$ and $p$, the adjoint variables $\mathbf{v}$ and $q$, and the parameter vector $\mathbf{m}$ are treated as variables that are independent of each other. Deriving the expressions for the gradient of the cost function $\mathcal{G}(\mathbf{m})$ can be achieved by computing the variations of the Lagrangian functional $\mathcal{L}$ with respect to the forward problem solution $(\mathbf{u}, p)$ and the adjoint variables $(\mathbf{v}, q)$, and setting them to zero. The remaining variation with respect to the parameter vector $\mathbf{m}$ gives the desired gradient expression.

Taking the directional derivative of $\mathcal{L}$ with respect to the adjoint variables $\mathbf{v}$ and $q$, and requiring it to vanish in the direction of all corresponding test functions $\hat{\mathbf{v}}$ and $\hat{q}$, yields:

$$
\mathcal{L}_{(\mathbf{v}, q)}(\mathbf{u}, p ; \mathbf{v}, q ; \mathbf{m})(\hat{\mathbf{v}}, \hat{q})=0
$$

Here one recovers the weak form of the general nonlinear Stokes equations (8), which is used to obtain the forward solution variables $\mathbf{u}$ and $p$.

Similarly, setting the directional derivatives of $\mathcal{L}$ with respect to the forward solution variables $\mathbf{u}$ and $p$ in the direction of all corre- 
sponding test functions $\hat{\mathbf{u}}$ and $\hat{p}$ to zero gives:

$$
\begin{array}{r}
\mathcal{L}_{(\mathbf{u}, p)}(\mathbf{u}, p ; \mathbf{v}, q ; \mathbf{m})(\hat{\mathbf{u}}, \hat{p})=\int_{\Omega_{\mathrm{obs}}} \frac{\partial \varphi}{\partial \mathbf{u}}(\mathbf{u})(\hat{\mathbf{u}})\left(\varphi-\varphi_{\mathrm{obs}}\right) d \mathbf{x} \\
+\int_{\Omega}(\mathbb{D}(\mathbf{u}) \dot{\varepsilon}(\hat{\mathbf{u}})): \dot{\boldsymbol{\varepsilon}}(\mathbf{v}) d \mathbf{x} \\
-\int_{\Omega} \hat{p} \nabla \cdot \mathbf{v} d \mathbf{x} \\
-\int_{\Omega} q \nabla \cdot \hat{\mathbf{u}} d \mathbf{x} \\
=0,
\end{array}
$$

where

$$
\mathbb{D}(\mathbf{u})=2 \eta(\mathbf{u})\left(\mathbb{I}+\left(\frac{1}{n}-1\right) \frac{\dot{\varepsilon}(\mathbf{u}) \otimes \dot{\varepsilon}(\mathbf{u})}{2 \dot{\varepsilon}_{\mathrm{II}}^{2}}\right)
$$

with $\mathbb{I}$ being the fourth-order identity tensor, and

$$
\begin{aligned}
\frac{\partial \varphi}{\partial \mathbf{u}}(\mathbf{u})(\hat{\mathbf{u}}) & =\frac{\hat{\dot{\varepsilon}}_{x y}\left(\dot{\varepsilon}_{x x}-\dot{\varepsilon}_{y y}\right)-\dot{\varepsilon}_{x y}\left(\hat{\dot{\varepsilon}}_{x x}-\hat{\dot{\varepsilon}}_{y y}\right)}{\left(\dot{\varepsilon}_{x x}-\dot{\varepsilon}_{y y}\right)^{2}+4 \dot{\varepsilon}_{x y}^{2}} \\
& =\frac{1}{\left(\dot{\varepsilon}_{x x}-\dot{\varepsilon}_{y y}\right)^{2}+4 \dot{\varepsilon}_{x y}^{2}} \frac{1}{2}\left(\mathbf{E}+\mathbf{E}^{T}\right): \hat{\dot{\varepsilon}}
\end{aligned}
$$

with

$$
\begin{aligned}
& \widehat{\dot{\varepsilon}}=\dot{\varepsilon}(\hat{\mathbf{u}}), \\
& \mathbf{E}=\mathbf{E}(\mathbf{u})=\left(\begin{array}{ccc}
-\dot{\varepsilon}_{x y} & \dot{\varepsilon}_{x x} & 0 \\
-\dot{\varepsilon}_{y y} & \dot{\varepsilon}_{x y} & 0 \\
0 & 0 & 0
\end{array}\right) .
\end{aligned}
$$

Equation (15) is referred to as the weak form of the adjoint equation and has to be solved for $\mathbf{v}$ and $q$, while $\mathbf{u}$ and $p$ indicate the forward problem solution corresponding to the parameters $\mathbf{m}$. Note that this equation is linear since the fourth-order tensor $\mathbb{D}(\mathbf{u})$ only depends on the converged forward solution and not on the adjoint variables $\mathbf{v}$ and $q$.

To complete the derivation of the gradient, the Lagrangian functional $\mathcal{L}$ is differentiated with respect to each material parameter. For example, the derivative with respect to the density parameter $\rho$ in some test direction $\hat{\rho}$ is given by:

$$
\mathcal{L}_{\rho}(\mathbf{u}, p ; \mathbf{v}, q ; \mathbf{m}) \hat{\rho}=-\int_{\Omega} \hat{\rho} \mathbf{g} \cdot \mathbf{v} d \mathbf{x}
$$

Differentiating with respect to the other parameters $\eta_{0}$ and $n$ yields the complete weak form of the gradient of the cost function $\mathcal{F}$. By isolating the test functions in the weak form, one finally obtains the equivalent strong form evaluated at $\mathbf{m}=\left(\rho, \eta_{0}, n\right)^{T}$ :

$$
\mathcal{G}(\mathbf{m})=\left(\begin{array}{c}
-\mathbf{g} \cdot \mathbf{v} \\
\eta_{0}^{-1} \boldsymbol{\tau}(\mathbf{u}): \dot{\varepsilon}(\mathbf{v}) \\
n^{-2}\left[\log \left(\dot{\varepsilon}_{0}\right)-\log \left(\dot{\varepsilon}_{\mathrm{II}}(\mathbf{u})\right)\right] \boldsymbol{\tau}(\mathbf{u}): \dot{\varepsilon}(\mathbf{v})
\end{array}\right) .
$$




\section{G.S. Reuber}

In this expression $(\mathbf{u}, p)$ denotes the solution of the forward Stokes equation (8) corresponding to the parameters $\mathbf{m}$, and $(\mathbf{v}, q)$ is the solution of the adjoint equation (15) corresponding to $(\mathbf{u}, p)$ and $\mathbf{m}$. Note that the first line of (21) is the gradient of the cost function with respect to $\rho$, the second line corresponds to $\eta_{0}$, and the third line to $n$. The gradient expression (21) is infinite-dimensional since it is defined on function spaces and therefore gives a parameter update in the form of a function that can be evaluated at any point in the domain, independent of the discretization.

Regardless of the parameter structure, equations (8) and (15) represent the weak form, and therefore can be readily used for a finite element discretization. Alternatively, the strong form of the adjoint system can be derived and discretized by finite differences. After isolating the test functions in the weak form using Greens first identity, the result is:

$$
\begin{aligned}
\nabla \cdot \mathbf{v} & =0 \\
-\nabla \cdot \hat{\boldsymbol{\sigma}} & = \begin{cases}\nabla \cdot\left(\frac{\varphi(\mathbf{u})-\varphi_{\mathrm{obs}}}{\left(\dot{\varepsilon}_{x x}-\dot{\varepsilon}_{y y}\right)^{2}+4 \dot{\varepsilon}_{x y}^{2}} \frac{1}{2}\left(\mathbf{E}+\mathbf{E}^{T}\right)\right) & \text { on } \Omega_{\mathrm{obs}}, \\
\mathbf{0} & \text { else }\end{cases}
\end{aligned}
$$

where

$$
\hat{\boldsymbol{\sigma}}=\hat{\boldsymbol{\sigma}}(\mathbf{v}, q)=\mathbb{D}(\mathbf{u}) \dot{\varepsilon}(\mathbf{v})-q \mathbf{I}
$$

and, assuming the measurements are not taken on the Earth's surface, the boundary conditions are

$$
\mathbf{v} \cdot \mathbf{n}=0, \quad \mathbf{T}(\hat{\boldsymbol{\sigma}} \mathbf{n})=\mathbf{0}
$$

The overall solution scheme employing the strong form of the adjoint equations (22) - (25) to compute the gradient (21) follows exactly the same path as the weak form solution outlined above.

\section{DISCRETIZATION}

\subsection{Forward problem}

We discretize the mass and momentum equations (1)-(2) using a staggered-grid finite difference scheme (Harlow \& Welch 1965; Gerya \& Yuen 2007) implemented in the 3D Stokes solver LaMEM (Kaus et al. 2016), which can handle visco-elasto-plastic materials. LaMEM has already been used as a forward problem solution tool in sampling-based inversion (Baumann et al. 2014) and gradient-based inversion (Reuber et al. 2018). The material advection is implemented using Lagrangian markers (Harlow \& Welch 1965). LaMEM is capable of handling a stable free surface boundary condition (Kaus et al. 2010; Duretz et al. 2011). For a given set of material parameters m, the discretized nonlinear equations are written in the form of

$$
\mathbf{r}(\mathbf{z}(\mathbf{m}), \mathbf{m})=\mathbf{0}
$$


where $\mathbf{r}$ and $\mathbf{z}=(\mathbf{u}, p)^{T}$ are the coupled residual and solution vectors, respectively. The residual vector is a direct result of discretizing the conservation equations (1) - (2) together with the boundary conditions (6) - (7) using the staggered-grid finite difference method (see e.g. Gerya \& Yuen 2007). The nonlinear system (26) is solved using the preconditioned Jacobian-free Newton-Krylov method with a line search as provided by the SNES nonlinear solver framework in PETSc (Balay et al. 2016):

$$
\begin{aligned}
\mathbf{A}^{-1} \mathbf{J}\left(\mathbf{z}_{k}\right) \delta \mathbf{z}_{k} & =-\mathbf{A}^{-1} \mathbf{r}\left(\mathbf{z}_{k}\right), \\
\mathbf{z}_{k+1} & =\mathbf{z}_{k}+\alpha \delta \mathbf{z}_{k}
\end{aligned}
$$

Here, $\delta \mathbf{z}$ is the iterative correction vector, $k$ is the iteration index, and $\alpha$ the line-search step length. The Jacobian matrix $\mathbf{J}=\partial \mathbf{r} / \partial \mathbf{z}$ is defined either analytically or implicitly by a matrix-vector product approximated by finite differences.

The preconditioning matrix $\mathbf{A}$ is given in the following block form:

$$
\mathbf{A}=\left(\begin{array}{ll}
\mathbf{K} & \mathbf{G} \\
\mathbf{D} & \mathbf{C}
\end{array}\right),
$$

where $\mathbf{K}$ denotes the stiffness matrix associated with the velocity variable, $\mathbf{D}$ is the velocity divergence matrix, and $\mathbf{G}$ is the pressure gradient matrix. The blocks $\mathbf{K}, \mathbf{D}$, and $\mathbf{G}$ are obtained by discretizing the conservation equations (1)-(2) using the effective viscosity (Picard linearization). The remaining block $\mathbf{C}$ comes from the diagonal approximation of the Schur complement based on the inverse viscosity scaling of the pressure mass matrix (see e.g. May \& Moresi 2008). To achieve optimal scalability of the Stokes solver, we employ a custom set of multigrid operators specifically designed for the staggered grid (Cai et al. 2014), which implies that the restriction and prolongation operators are not transposes of each other. The corresponding Galerkin coarsening process is implemented in the PETSc multigrid framework (Balay et al. 2016). For more details the reader is referred to Kaus et al. (2016).

\subsection{Discrete adjoint gradient}

In a finite element context, the strong form gradient equation (21) must be discretized using the appropriate weak form. In practice, this involves selecting a set of test functions that corresponds to the assumed parameter variation (bilinear, piecewise constant, etc.), and switching to the integral form, similar to equation (20). For example, in this work we partition the domain into a set of non-overlapping subdomains occupied by material phases. Within each subdomain we assume a piecewise constant approximation for the material parameters (see Fig. 2). In any case, the number of entries in the discrete gradient vector is controlled by the number of parameters used by the selected material parameter test functions (one entry per parameter, which is 6 for the case shown in Fig. 2).

In a finite difference context, it is not guaranteed that equation (21) is automatically consistent with the finite difference stencil used for the forward problem, because of the involved interpolation. For a pointwise parameter discretization, equation (21) can be used directly. However, other types of parameter discretizations, such as piecewise constant (Fig. 2), require computing a weighted sum of derivatives 


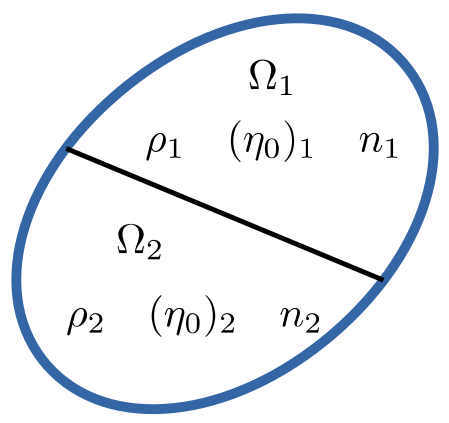

Figure 2. Material parameters discretization scheme. Material phases are occupying non-overlapping subdomains. All phase properties are assumed to be constant within each phase subdomain.

similar to (21) for each discrete material parameter. The choice of these weights depends on the particular discretization and must be made carefully in order to be consistent with interpolation and parameter discretization. To avoid these complications, it becomes convenient to select the discretization first, and then derive the adjoint equations depending on this discretization. This alternative way to obtain the adjoint gradient is described in the following section.

\subsection{Adjoint equations}

The adjoint formulation can also be derived purely algebraically, after selecting the appropriate discretization of the forward problem and material parameter field, in a finite-dimensional sense. This formulation is not limited to finite differences, but can also be used in the finite element context. Denoting the solution of the discrete forward problem by $\mathbf{z}$, which depends on the set of material parameters $\mathbf{m}$, one can define an objective function in a general form:

$$
F=F(\mathbf{z}(\mathbf{m}))
$$

In this work, we minimize the misfit between the computed principal strain directions $\varphi$ and the measured ones $\varphi_{\text {obs }}$ :

$$
F=F\left(\varphi_{\mathrm{obs}}, \boldsymbol{\varphi}(\dot{\varepsilon}(\mathbf{u}(\mathbf{m})))\right)=\frac{1}{2}\left\|\varphi-\varphi_{\mathrm{obs}}\right\|^{2}
$$

where $\|\cdot\|$ denotes the Euclidean norm.

The gradients of the objective function with respect to the model parameters can be computed using the following two-step procedure (e.g. Giles \& Pierce 2000):

$$
\begin{aligned}
\boldsymbol{\psi} & =\left(\mathbf{J}^{T}\right)^{-1}\left(\frac{\partial F}{\partial \mathbf{z}}\right)^{T}, \\
\frac{d F}{d \mathbf{m}} & =-\boldsymbol{\psi}^{T} \frac{\partial \boldsymbol{r}}{\partial \mathbf{m}} .
\end{aligned}
$$

Here $\mathbf{J}$ denotes the Jacobian matrix that is already used by the Newton forward solver and can be reused for the adjoint equation (32) (see e.g. 
Worthen et al. 2014), which makes this approach computationally particularly appealing. The partial derivative of the residual with respect to the parameters can be computed analytically, or approximated by finite differences (at the cost of accuracy and efficiency):

$$
\frac{\partial \mathbf{r}}{\partial m_{i}} \approx \frac{\mathbf{r}\left(\mathbf{m}+h \mathbf{e}_{i}\right)-\mathbf{r}(\mathbf{m})}{h}
$$

In this equation, $h$ is a small perturbation parameter and $\mathbf{e}_{i}$ is the $i$-th unit vector. Since every material parameter, in general, only influences very few residual entries, it can be practical to handle the discrete gradient (33) as a sparse matrix-vector product, which can be computed on the fly in a matrix-free manner.

The remaining derivative of the objective function with respect to the forward solution vector can be expanded using the chain rule as follows:

$$
\frac{\partial F}{\partial \mathbf{z}}=\frac{\partial F}{\partial \varphi} \frac{\partial \varphi}{\partial \dot{\varepsilon}} \frac{\partial \dot{\varepsilon}}{\partial \mathbf{u}}
$$

where

$$
\frac{\partial F}{\partial \varphi}=\varphi-\varphi_{\mathrm{obs}}
$$

and

$$
\frac{\partial \varphi}{\partial \dot{\varepsilon}}=\frac{1}{\left(\dot{\varepsilon}_{x x}-\dot{\varepsilon}_{y y}\right)^{2}+4 \dot{\varepsilon}_{x y}^{2}}\left(-\dot{\varepsilon}_{x y}, \dot{\varepsilon}_{x y}, \dot{\varepsilon}_{x x}-\dot{\varepsilon}_{y y}\right)
$$

The last term $\frac{\partial \dot{\varepsilon}}{\partial \mathbf{u}}$ is discretization-dependent. For the staggered-grid finite difference discretization, this derivative is summarized in appendix A. For a finite element discretization, it has a structure similar to the strain operator matrix.

The computational steps include solving the nonlinear forward problem (27)-(28), followed by one adjoint linear solve (32), and calculating the gradients according to (33). The overall numerical cost is essentially independent of the number of material parameters.

The computed gradients can be used in any gradient-based inversion framework. In LaMEM, we can use both the TAO optimization library, which is provided through PETSc, and a simple steepest descent algorithm. The TAO BLMVM optimization tool applies a BFGS approximation of the Hessian (H) and the Moré-Thuente line search (Munson et al. 2012) to update the parameter vector with a quasi-Newton scheme:

$$
\mathbf{m}_{l+1}=\mathbf{m}_{l}-\beta \mathbf{H}_{l}^{-1} \frac{d F}{d \mathbf{m}}
$$

where $l$ is the inversion iteration counter and $\beta$ is a line-search parameter. 


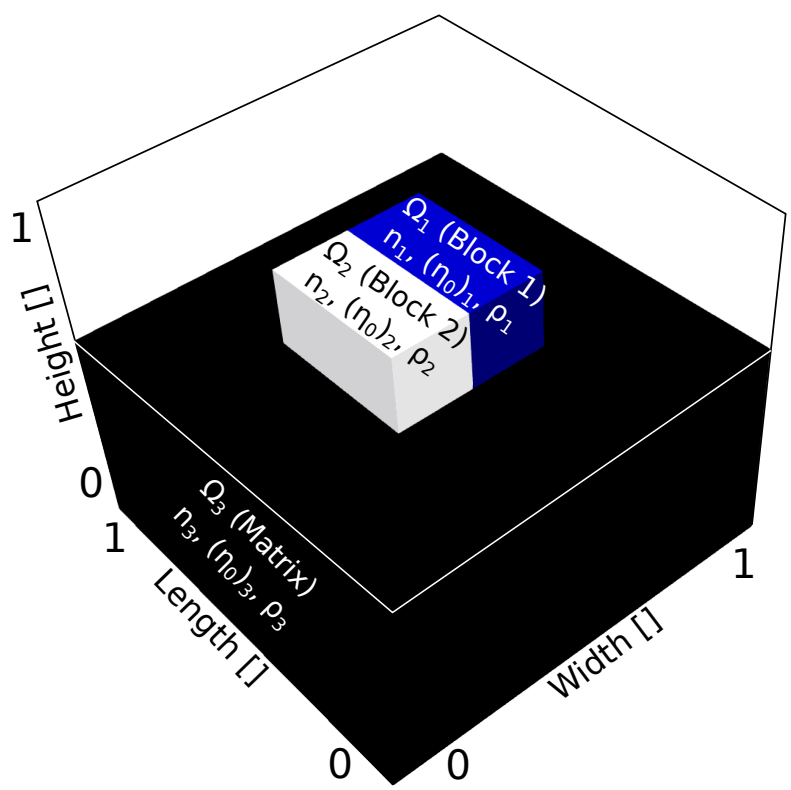

Figure 3. Model setup. A denser and more viscous cube sinks into a matrix. The cube is centered in the middle of the domain, which consists of two equally sized parts $\Omega_{1}$ and $\Omega_{2}$. The less viscous and less dense matrix is denoted by $\Omega_{3}$. Each phase is described by a constant power-law exponent $n_{i}$, constant reference viscosity $\eta_{i}$ and a constant density $\rho_{i}$. The synthetic data for the inversions in sections $4.3-4.6$ is taken from a near-surface observation point $P$ with coordinates $\mathbf{x}_{P}=(0.6,0.5,0.99)^{T}$.

\section{RESULTS}

\subsection{Model setup}

We test the proposed adjoint extension with PSD constraints using a synthetic falling block setup. The model consists of a higher viscosity and denser cubic block, with a side length of 0.5 , placed in the center of a unit cube domain (see Fig. 3). The domain is filled by a lower viscosity and lighter matrix, which causes the block to sink. The domain is discretized by $32 \times 32 \times 32$ cells of the staggered-grid finite difference and has free slip boundary condition on all sides of the model domain.

We test both linear and nonlinear (power-law) rheology scenarios. The non-dimensional reference material parameters are listed for both linear and nonlinear cases in Table 1 . The stiff block inclusion is divided into two equally sized parts, referred to in the following as Block 1 and Block 2, as shown in Fig. 3. In the subsequent inversion tests we keep the rheology of the matrix and Block 2 fixed. The material parameters of Block 1 are perturbed and synthetically inverted for, using the adjoint gradients of the observed PSD field. To mimic the sparsity of observations in natural applications, we only use one PSD measurement at a single near-surface point, which is referred to as $P$ in the following sections, with coordinates $\mathbf{x}_{P}=(0.6,0.5,0.99)^{T}$. Since we keep the material parameters constant within the phases, the resulting discrete adjoint gradient vector has very few entries (see section 3.2). 
Table 1. Reference linear and nonlinear material parameters.

\begin{tabular}{lccccc}
\hline Phase & $\rho$ & $\eta$ & $\eta_{0}$ & $\varepsilon_{0}$ & $\mathrm{n}$ \\
\hline Matrix & 1 & 1 & 1 & $10^{-6}$ & 2 \\
Block 1 and 2 & 2 & 100 & 100 & $10^{-6}$ & 2 \\
\hline
\end{tabular}

\subsection{Forward result}

Figs. 4 - 6 show typical results of the forward problem. For a homogeneous block rheology, the principal stress orientation forms a concentrically symmetric pattern around a vertical axis passing through the block center (Fig. 4a). The computed near-surface Stokes velocity field (Fig. 4b) shows a pattern correlating with a downward block motion. The principal stress orientation visualized as a scalar angle field is shown at different depths in Fig. 5 for the homogeneous rheology case, and in Fig. 6 for the heterogeneous rheology case (i.e. Block 1 has a different rheology compared to Block 2). As expected, the homogeneous case is characterized by the symmetric PSD orientation pattern at all depths, whereas for the heterogeneous case this symmetry is lost.

\subsection{Adjoint gradient test}

Before performing an actual gradient-based inversion, it is necessary to verify the adjoint-based gradients numerically. This can be done by comparing the adjoint gradients with forward finite differences of the cost function with respect to the material parameters $m_{i}$ :

$$
\frac{\partial F}{\partial m_{i}} \approx \frac{F\left(\mathbf{z}\left(\mathbf{m}+h \mathbf{e}_{i}\right)\right)-F(\mathbf{z}(\mathbf{m}))}{h}
$$
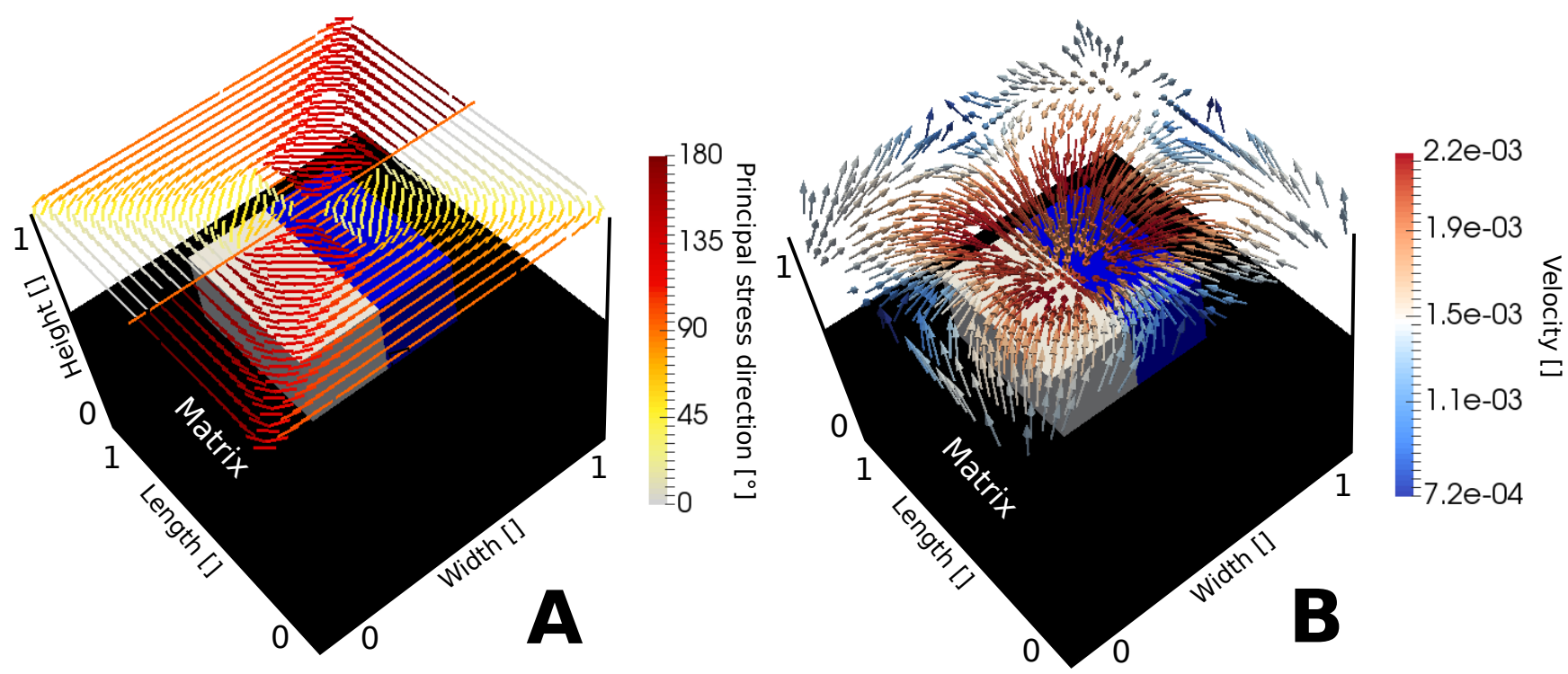

Figure 4. Forward modeling results of the principal stress directions w.r.t. $x$-axis (A) and the velocity field (B) for the homogeneous cube. 

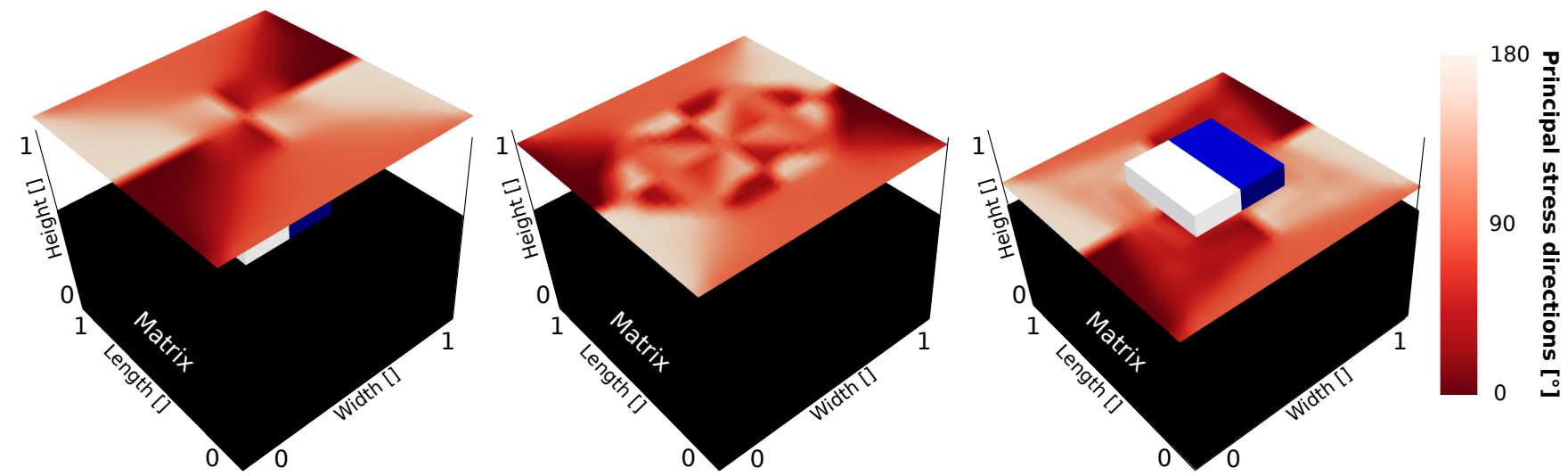

Figure 5. Stress direction field in three planes at different depths $(1,0.8,0.6)$ in case of a homogeneous cube.

For sufficiently small $h$, the right-hand side should be close to the gradient obtained using the adjoint equations. This test amounts to obtaining the forward problem solution once unperturbed and additionally once per perturbed material parameter $m_{i}$ per perturbation size $h$.

In this test we use a homogeneous setup with linear rheology, and evaluate cost function (equation (31)) at a near-surface observation point $P$. As the observed orientation is not important for this test, we use a random value for $\varphi_{\text {obs }}$ in equation (31). By perturbing the density and viscosity parameters of Block 1, we approximate the corresponding components of the gradient vector using the finite differences (equation (39)), and compare those with the output of the adjoint-based gradient (equation (33)). The results of the gradient test convergence for a decreasing perturbation parameter $h$ are shown in Fig. 7. Clearly, the adjoint method reproduces the finite-difference gradient for a large range of perturbations. The error decreases with decreasing $h$ down to about the square root of machine precision, after which it increases again which is a well-known shortcoming of numerical differentiation (see e.g. Engl et al. 1996).
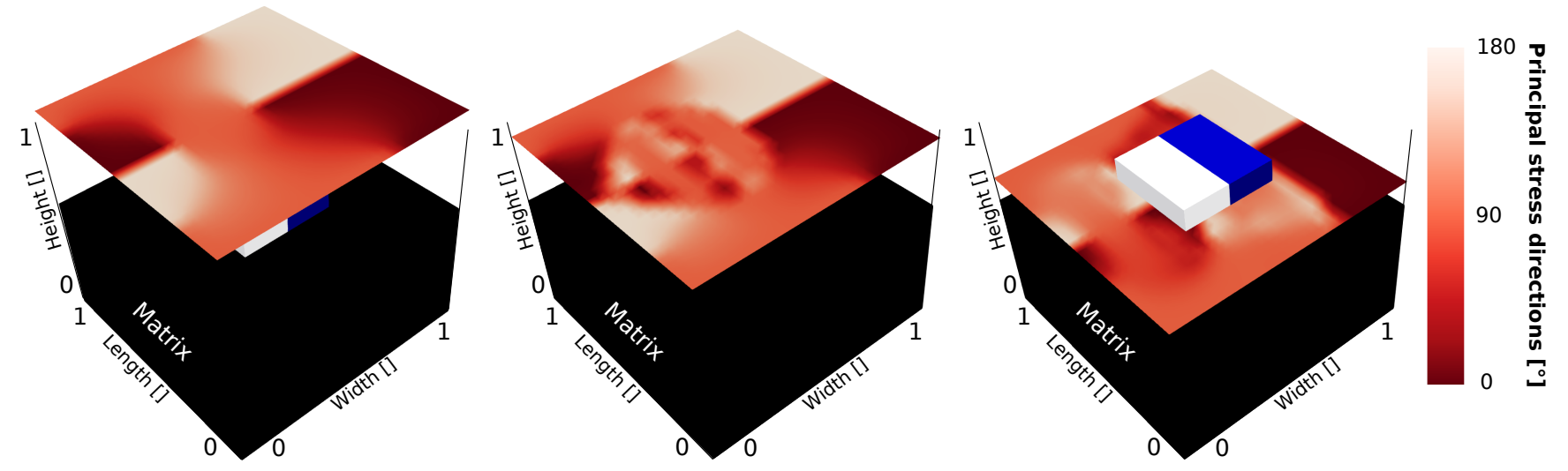

Figure 6. Stress direction field in three planes at different depths $(1,0.8,0.6)$. Block 1 has the same rheology as the Matrix. 


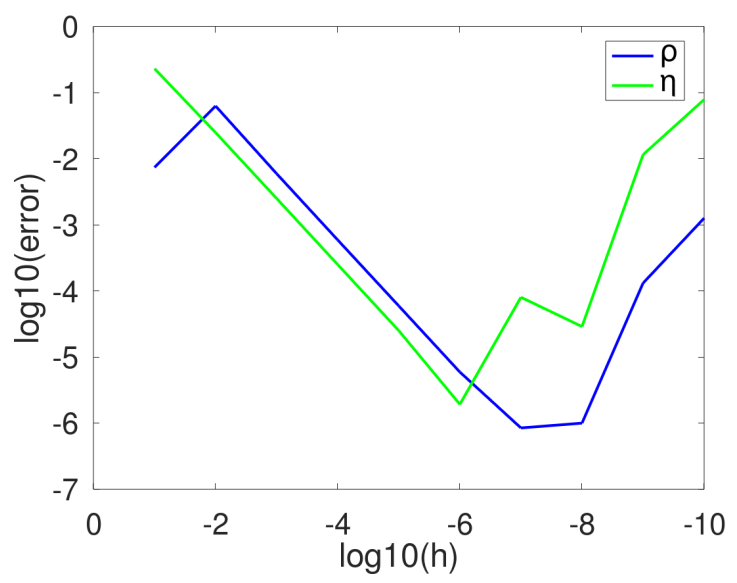

Figure 7. Numerical verification of the gradient of $\rho$ of Block 1 (blue line) and $\eta$ of Block 1 (green line) computed using the adjoint equation. The $x$-axis shows different values for the perturbation $h$, whereas the $y$-axis indicates the logarithmic value of the normalized difference between the gradient computed using finite differences (39) and the gradient obtained through the adjoint equation (33). The test is performed at point $P$ for a linear setup with the rheological parameter of Block 1 equal to the one of the Matrix.

\subsection{Inversion result}

Here we present a set of simple computations that illustrate the behavior of the adjoint-based inversion using PSD constraints. All computations start with a synthetic velocity and PSD orientation field. Next, one of the following parameters are perturbed: density $\rho$, linear viscosity $\eta$, or power-law reference viscosity $\eta_{0}$ of Block 1 . As before, the PSD observations and vertical velocity, respectively, at point $P$ are used to formulate the cost function.

Fig. 8 shows the convergence of the gradient-based inversion for the linear viscous case. Here we perform two separate single-parameter inversions, one for viscosity and one for density. The red lines in the plots in Fig. 8 indicate the convergence of the respective material parameter from the initial guess to the inverted one. The results show that both parameters converge to the synthetic value within a few inverse iterations.

Similar to the previous case, we perform two inversions for a nonlinear power-law rheology. Fig. 9 shows the inversion results for density and power-law reference viscosity. Again the red lines in the plots indicate the convergence of the material parameter from the initial guess to the inverted one. As in the linear viscous case, convergence is achieved very rapidly.

\subsection{Sensitivity analysis}

In general, inverse problems governed by elliptic partial differential equations like the Stokes equation are often ill-posed (see e.g. Engl et al. 1996). The inverse problem addressed in this work suffers from this aspect as well since only very few observations can be taken into account - only one point in the synthetic examples in our test cases, and very few points in real-world examples. This leads to non-unique minimizers 

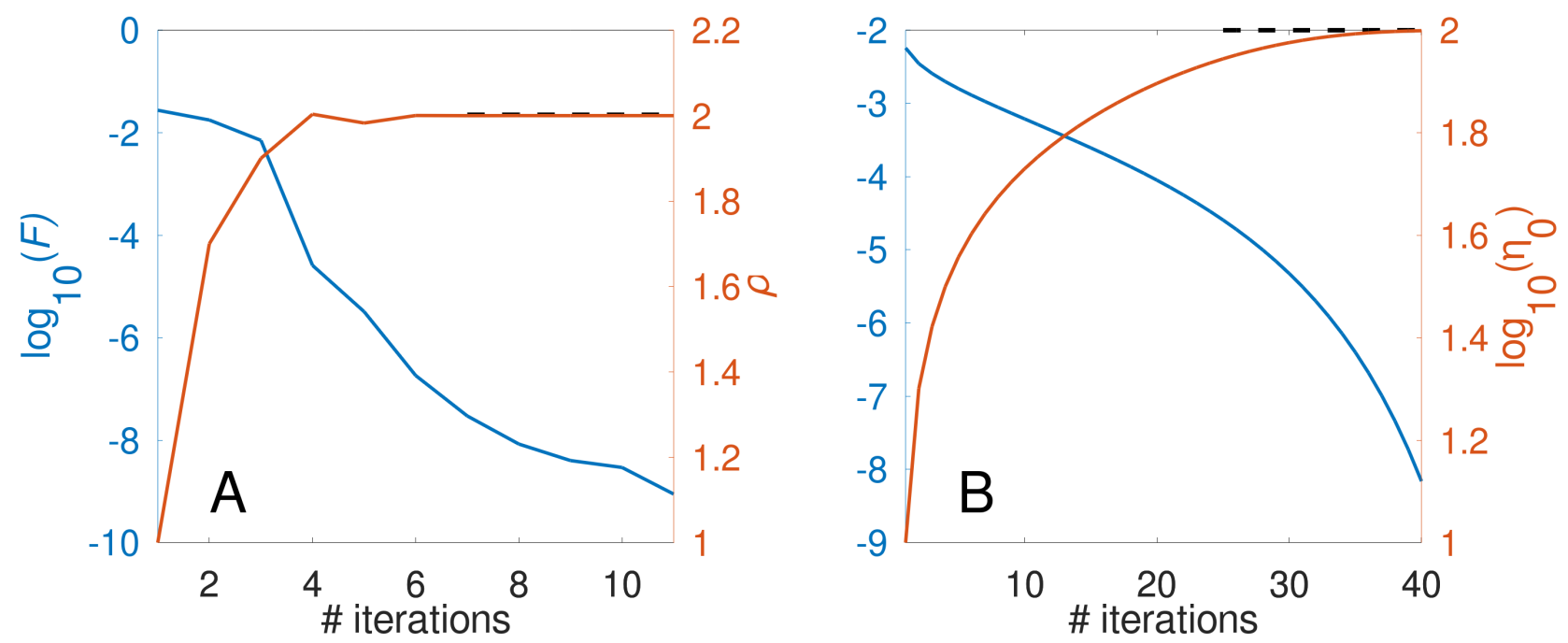

Figure 8. Result of inversion for linear parameters, where the $x$-axis indicates the number of iterations and the left $y$-axis shows the logarithm of the cost function value $F$. The dashed black line indicates the true synthetic value. A: Convergence history of the density. B: Convergence history of the linear viscosity.

of the cost function for the subsurface structure. Nevertheless, the gradients of the cost functions constrained by PSD or velocity observations can still give an insight into the physical response of the model.

In this context, it becomes interesting to compare the sensitivity of both constraint types (PSD and velocity) to the parameter variations. A straightforward approach is to perform a grid-search sampling. We discretize the two cost functions with a regular grid in the density and linear viscosity parameter space and evaluate the cost function at each parameter combination. The result shows that both cost functions have
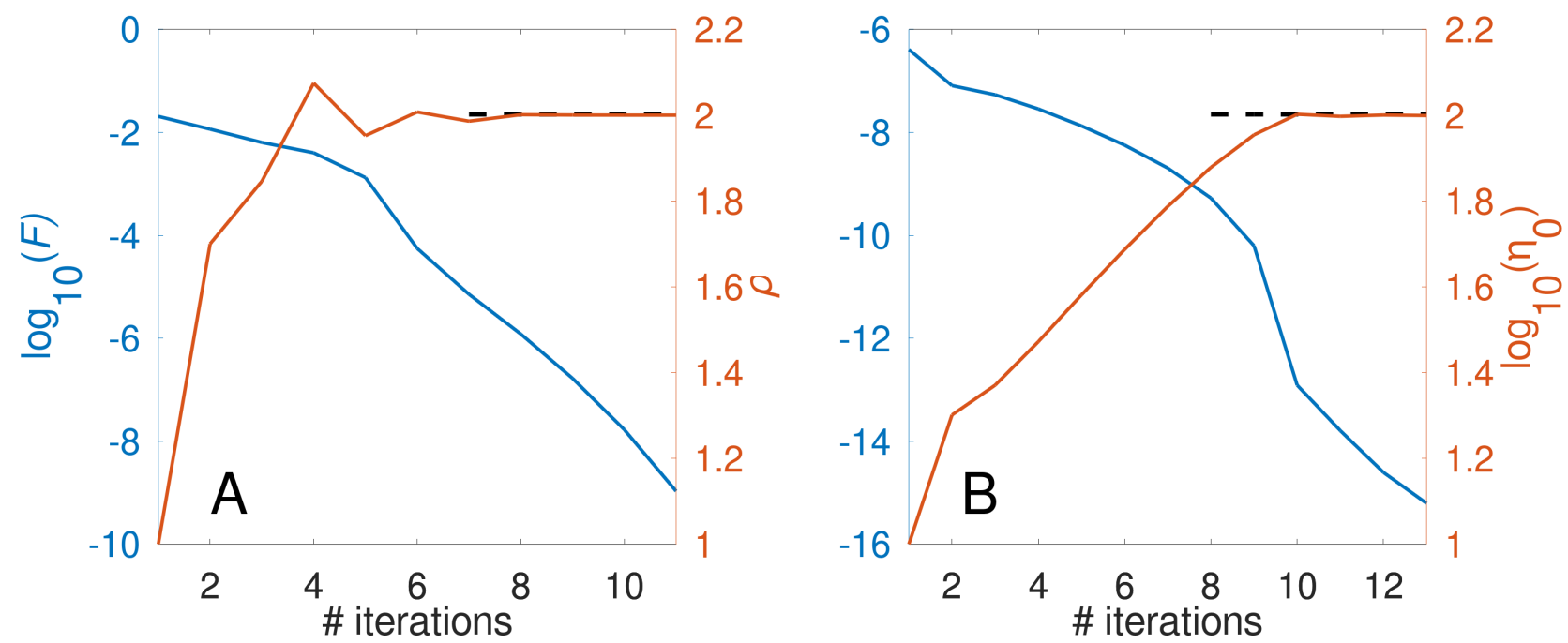

Figure 9. Result of inversion for nonlinear parameters, where the $x$-axis indicates the number of iterations and the left $y$-axis shows the logarithm of the cost function value $F$. The dashed black line indicates the true synthetic value. A: Convergence history of the density. B: Convergence history of the power-law reference viscosity. 

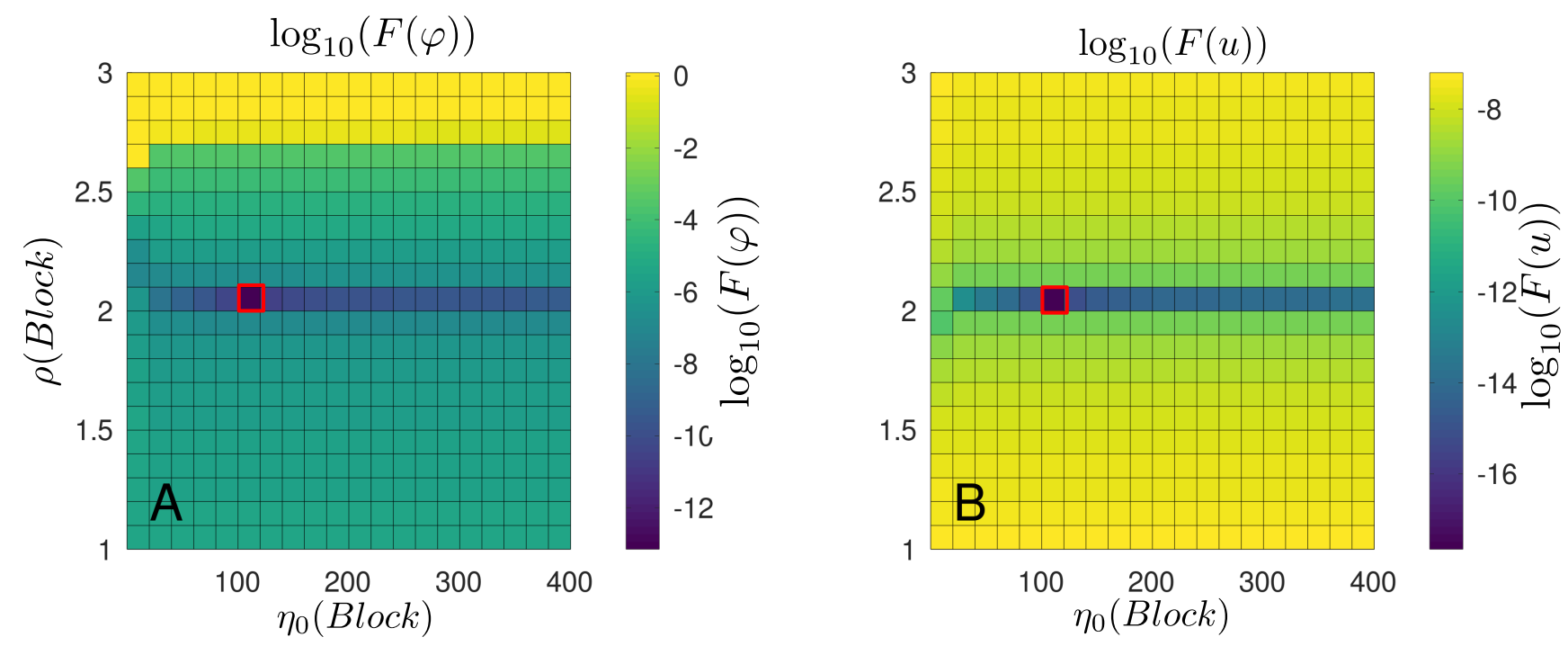

Figure 10. Cost function sensitivity comparison by the grid search for PSD constraint (A), and velocity constraint (B). $x$-axis represents the viscosity $\eta$, $y$-axis represents the density $\rho$. The colors indicate the values of the cost functions. The red squares indicate the synthetic parameters and thus define a global minimum of the cost function.

multiple local minima in the parameter space (Fig. 10). The shapes of the cost functions are very similar, since both the velocity and the PSD are more sensitive to the density than to the viscosity. However, the values for the PSD-based cost function are much higher, which indicates a generally higher sensitivity of the PSD to a change in the material parameters for this particular computation.

\subsection{Geodynamic sensitivity kernels}

Another approach to gain insight into the sensitivity of the measurements to the material parameters is to visualize the spatial distribution of the gradients, sometimes referred to as sensitivity kernels in seismic inversions (c.f. Tromp et al. 2005). Here, one takes the PSD observation at one (or multiple) point(s) and calculates the partial derivatives with respect to the material parameters, which are, in this case, defined in each grid point. The resulting field indicates in which regions of the domain a change of the material parameters has an effect on the measured data. The sensitivity kernel defined here is thus not to be confused with the gradient of the cost function.

The sensitivity kernels can be obtained in the same way as the gradients in section 3.3 but the function $F$ needs to be replaced by

$$
\hat{F}(\mathbf{m})=\varphi(\mathbf{m})\left(\mathbf{x}_{P}\right)=\varphi_{P}
$$

where $\varphi_{P}$ is the PSD at some point $\mathbf{x}_{P}$, corresponding to the parameters $\mathbf{m}$. Similarly, one can compute the sensitivity kernels for the $z$-velocity by adjusting equation (40) accordingly. The derivatives $\frac{\partial \hat{F}}{\partial m_{i}}\left(m_{i}\right)$ are shown in Fig. 11 for the velocity in the left column and the PSD in the right column, both measured at the observation point $P$. In order to obtain these kernels only one forward solve and two adjoint solves are required.

The shapes of the kernels are very different, which means that for this specific setup, the PSD is influenced by other parts of the domain 
than the velocity. In particular, the PSD seems to be sensitive to changes of the material parameters in a significantly larger region compared to the velocity. This different shape of the kernels may allow for a more precise determination of the shape or magnitude of an anomaly than only using one of the observables. This makes the PSD an interesting observation in real applications to get additional insights into the model.

\section{CONCLUDING REMARKS}

In this work, we present the adjoint method for efficient computation of gradients using principal stress directions as observations. These gradients can be used in inversion methods to infer material parameters of interest inside the Earth's crust and lithosphere, such as density or viscosity. Constraining these solely from surface observations results in an ill-posed inverse problem, which can potentially be improved by including as many observations as possible. Even though PSD measurements are costly and difficult to obtain and thus only sparsely available (e.g. Heidbach et al. 2018), they can enhance an inversion framework that is solely based on GPS velocities (e.g. Kreemer et al. 2003).

We demonstrate two ways of computing the adjoint gradients. The first approach is formally independent of the discretization of the forward and the adjoint problem, while the second approach derives the adjoint formulation purely algebraically, after the appropriate decisions about the discretization of the conservation equations and parameter fields have been made.

We present a grid-search-based sensitivity analysis for the cost functions employing both PSD and surface (plate) velocities as constraints. The results show that the shapes of both cost functions are very similar. However, the sensitivity of the PSD is higher to smaller variations in the parameters for the simplified example presented in this work, which may help to accelerate the convergence of the inversion.

We also suggest a way to analyze the sensitivity of observations to spatial variation of the material parameters, commonly referred to as sensitivity kernels. Whereas such kernels are well-known in the context of full waveform inversion, it has not yet been used in a geodynamic context. Results for our test case show that the spatial sensitivity of the PSD constraint extends to much larger domains compared to the velocity, which makes measurements of the PSD additionally interesting.

In general, preliminary results based on the synthetic computations are encouraging and suggest that incorporation of PSD in the computational framework helps improve the robustness and quality of geodynamic inversions. More work is required to test this for natural cases. 

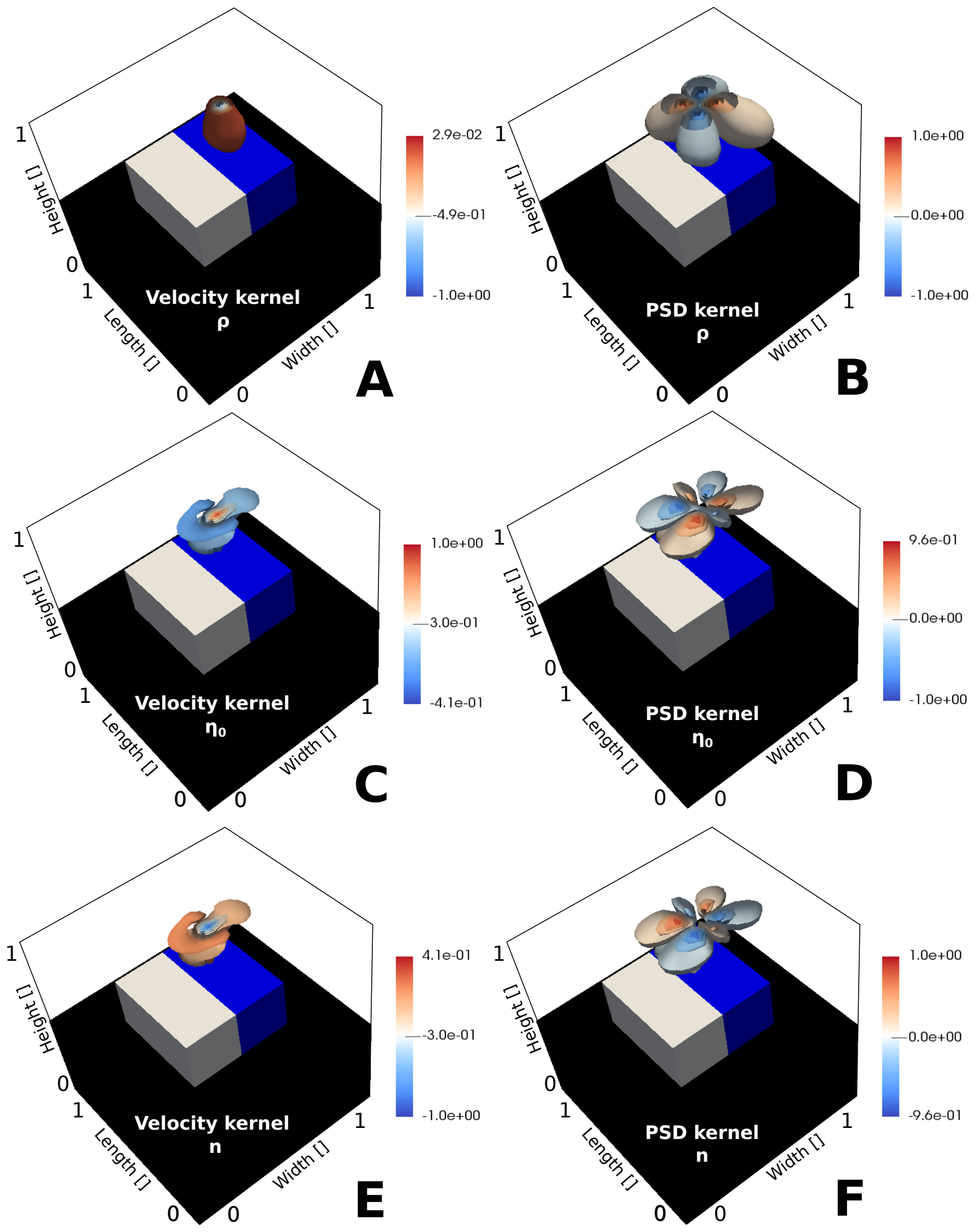

Figure 11. From top to bottom, contour lines of the pointwise sensitivity kernels with respect to the density, reference viscosity, and power-law exponent are shown for the velocity $u_{z}$ in the left column (A, C, E), and the PSD in the right column (B, D, F). In all cases, the measurements were taken at the near-surface observation point $P$. The values of the kernels are normalized. The sensitivity kernel can be seen as the derivative of the forward problem operator (contrary to the cost function) with respect to the material parameter in every grid point. 


\section{ACKNOWLEDGMENTS}

The authors gratefully acknowledge the computing time granted on the supercomputer Mogon 2 at Johannes Gutenberg University Mainz (hpc.uni-mainz.de). GR acknowledges support from DFG grant KA3367/4 and the Max-Planck Graduate Center Mainz. BK acknowledges support from the ERC consolidator grant 771143 (MAGMA). MH, BK and AP acknowledge the Computational Sciences Mainz framework. The authors additionally acknowledge Karina Koval for careful proofreading.

\section{REFERENCES}

Balay, S., Abhyankar, S., Adams, M., Brown, J., Brune, P., Buschelman, K., Dalcin, L., Eijkhout, V., Gropp, W., Kaushik, D., Knepley, M., Curfman-McInnes, L., Rupp, K., Smith, B., Zampini, S., Zhang, H., \& Zhang, H., 2016. PETSc Users Manual.

Baumann, T. S., Kaus, B. J., \& Popov, A. A., 2014. Constraining effective rheology through parallel joint geodynamic inversion, Tectonophysics, 631, $197-211$.

Bertsekas, D., 2014. Constrained optimization and Lagrange multiplier methods, Academic press.

Cai, M., Nonaka, A., Bell, J. B., Griffith, B. E., \& Donev, A., 2014. Efficient variable-coefficient finite-volume stokes solvers, Communications in Computational Physics, 16(5), 1263-1297.

Duretz, T., May, D. A., Gerya, T. V., \& Tackley, P. J., 2011. Discretization errors and free surface stabilization in the finite difference and marker-in-cell method for applied geodynamics: A numerical study, Geochemistry, Geophysics, Geosystems, 12(7), n/a-n/a.

Engl, H., Hanke, M., \& Neubauer, A., 1996. Regularization of inverse problems, Kluwer Academic Publishers, Dordrecht.

Fichtner, A., 2011. Full Seismic Waveform Modelling and Inversion, Springer Berlin Heidelberg.

Gere, J., 2004. Mechanics of materials, Thomson Learning, 6th edn.

Gerya, T. V. \& Yuen, D. A., 2007. Robust characteristics method for modelling multiphase visco-elasto-plastic thermo-mechanical problems, Physics of the Earth and Planetary Interiors, 163(1-4), 83-105.

Giles, M. B. \& Pierce, N. A., 2000. An introduction to the adjoint approach to design, Flow, turbulence and combustion, 65(3-4), 393-415.

Harlow, F. H. \& Welch, J. E., 1965. Numerical calculation of time-dependent viscous incompressible flow of fluid with free surface, Physics of Fluids, 8(12), $2182-2189$.

Heidbach, O., Rajabi, M., Cui, X., Fuchs, K., Müller, B., Reinecker, J., Reiter, K., Tingay, M., Wenzel, F., Xie, F., et al., 2018. The world stress map database release 2016: Crustal stress pattern across scales, Tectonophysics, 744, 484-498.

Horbach, A., Bunge, H.-P., \& Oeser, J., 2014. The adjoint method in geodynamics: derivation from a general operator formulation and application to the initial condition problem in a high resolution mantle circulation model, GEM - International Journal on Geomathematics, 5, $163-194$.

Ismail-Zadeh, A. T., Korotkii, A. I., Naimark, B. M., \& Tsepelev, I. A., 2003. Three-dimensional numerical simulation of the inverse problem of thermal convection, Computational Mathematics and Mathematical Physics, 43(4), 581-599.

Kaus, B., Popov, A., Baumann, T., Psk, A., Bauville, A., Fernandez, N., \& Collignon, M., 2016. Forward and inverse modelling of lithospheric deformation 
on geological timescales, in NIC Symposium 2016 - Proceedings, vol. 48 of NIC Series, pp. 299-307, eds Binder, K., Mller, M., \& Schnurpfeil, A., Forschungszentrum Jlich, Jlich.

Kaus, B. J., Mühlhaus, H., \& May, D. A., 2010. A stabilization algorithm for geodynamic numerical simulations with a free surface, Physics of the Earth and Planetary Interiors, 181(1-2), 12-20.

Kreemer, C., Holt, W. E., \& Haines, A. J., 2003. An integrated global model of present-day plate motions and plate boundary deformation, Geophysical Journal International, 154(1), 8-34.

May, D. \& Moresi, L., 2008. Preconditioned iterative methods for stokes flow problems arising in computational geodynamics, Physics of the Earth and Planetary Interiors, 171, $33-47$.

Munson, T., Sarich, J., Wild, S., \& Bensonand, S., 2012. TAO 2.0 User Manual, Argonne National Laboratory.

Ratnaswamy, V., Stadler, G., \& Gurnis, M., 2015. Adjoint-based estimation of plate coupling in a non-linear mantle flow model: theory and examples, Geophysical Journal International, 202(2), 768-786.

Reuber, G. S., Popov, A. A., \& Kaus, B. J., 2017. Deriving scaling laws in geodynamics using adjoint gradients, Tectonophysics, 746, $352-363$.

Reuber, G. S., Kaus, B. J. P., Popov, A. A., \& Baumann, T. S., 2018. Unraveling the physics of the yellowstone magmatic system using geodynamic simulations, Frontiers in Earth Science, 6.

Tarantola, A., 2005. Inverse Problem Theory and Methods for Model Parameter Estimation, Society for Industrial and Applied Mathematics.

Tröltzsch, F., 2010. Optimal Control of Partial Differential Equations: Theory, Methods and Applications, vol. 112, American Mathematical Society Graduate Studies in Mathematics.

Tromp, J., Tape, C., \& Liu, Q., 2005. Seismic tomography, adjoint methods, time reversal and banana-doughnut kernels, Geophysical Journal International, 160(1), 195-216.

Worthen, J., Stadler, G., Petra, N., Gurnis, M., \& Ghattas, O., 2014. Towards adjoint-based inversion for rheological parameters in nonlinear viscous mantle flow, Physics of the Earth and Planetary Interiors, 234, 23-34.

Zoback, M., 2010. Reservoir Geomechanics, Cambridge University Press.

\section{APPENDIX A: STAGGERED-GRID FINITE DIFFERENCE DISCRETIZATION OF STRAIN RATE GRADIENT TERM}

Assuming Anderson's model and selecting the coordinate system such that the $x$ - and $y$-axes span the horizontal plane leaves only three strain rate components relevant for computing the principal horizontal stresses (strain rates), which can be packed in a vector as follows:

$$
\dot{\varepsilon}=\left(\dot{\varepsilon}_{x x}, \dot{\varepsilon}_{y y}, \dot{\varepsilon}_{x y}\right)^{T}
$$

The staggered-grid finite difference stencil required to interpolate these components is shown in Fig. A1. Additionally assuming that PSD observations $\varphi_{\text {obs }}$ are defined in the cell centers eliminates the need to interpolate the normal strain rates $\dot{\varepsilon}_{x x}$ and $\dot{\varepsilon}_{y y}$. The single shear strain rate component $\dot{\varepsilon}_{x y}$ can be interpolated to the cell center from the edges. With these interpolation schemes, there are in total $12 x$-and $y$-velocity components that influence the relevant strain rates. By packing them in a small vector $\mathbf{u}$, in the order shown in Fig. A1, we can 
express the interpolation in the following matrix form:

$$
\begin{aligned}
& \dot{\boldsymbol{\varepsilon}}=\mathbf{B} \mathbf{u}, \\
& \mathbf{u}=\left(u_{1}, u_{2}, \ldots u_{12}\right)^{T} .
\end{aligned}
$$

Here the interpolation matrix is given by:

$$
\begin{aligned}
& \mathbf{B}=\left(\begin{array}{ccccc}
-b_{x} & b_{x} & 0 & 0 & \\
0 & 0 & -b_{y} & b_{y} & \cdots \\
b_{y b}-b_{y f} & b_{y b}-b_{y f} & b_{x b}-b_{x f} & b_{x b}-b_{x f}
\end{array}\right.
\end{aligned}
$$

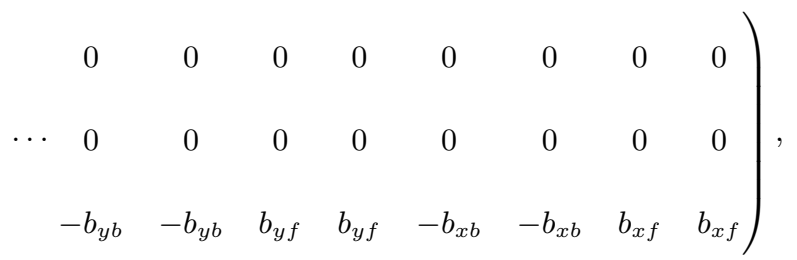

with

and

$$
\begin{aligned}
& b_{x}=\frac{1}{\Delta x_{i}}, \quad b_{x b}=\frac{1}{8 \Delta x_{b}}, \quad b_{x f}=\frac{1}{8 \Delta x_{f}}, \\
& b_{y}=\frac{1}{\Delta y_{j}}, \quad b_{y b}=\frac{1}{8 \Delta y_{b}}, \quad b_{y f}=\frac{1}{8 \Delta y_{f}},
\end{aligned}
$$

$$
\begin{aligned}
\Delta x_{b} & =\frac{1}{2}\left(\Delta x_{i-1}+\Delta x_{i}\right), \quad \Delta x_{f}=\frac{1}{2}\left(\Delta x_{i}+\Delta x_{i+1}\right), \\
\Delta y_{b} & =\frac{1}{2}\left(\Delta y_{j-1}+\Delta y_{j}\right), \quad \Delta y_{f}=\frac{1}{2}\left(\Delta y_{j}+\Delta y_{j+1}\right) .
\end{aligned}
$$

Hence the required gradient term is simply given by:

$$
\frac{\partial \dot{\varepsilon}}{\partial \mathbf{u}}=\mathbf{B}
$$




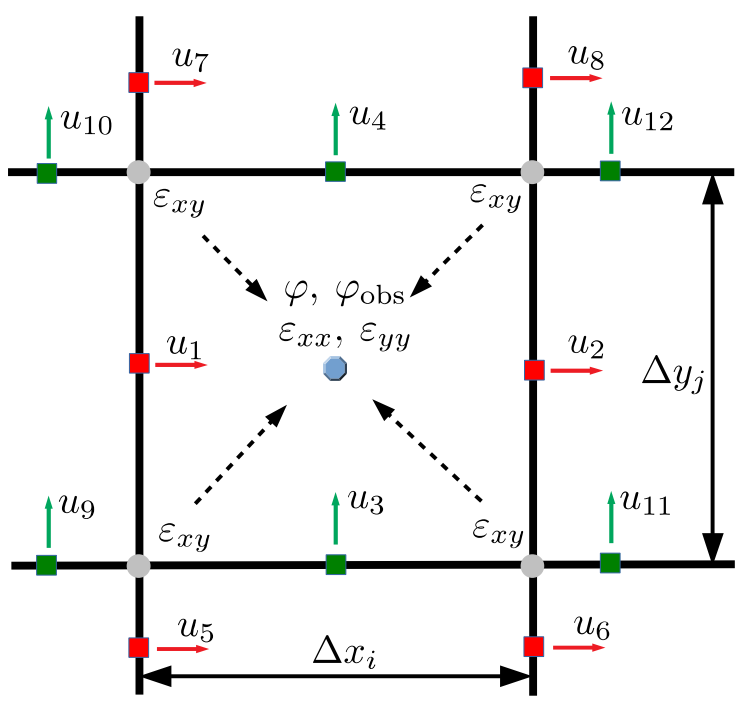

Figure A1. Staggered-grid finite difference discretization stencil for the $\frac{\partial \dot{\varepsilon}}{\partial \mathbf{u}}$ term. Shown is a cross-section of a 3D cell in the horizontal $(x-y)$ plane. Circles represent middle points of the edges, squares represent centers of the faces. 